\title{
A REMARK ON REPRESENTATIONS OF GROUPS*
}

\section{TADASI NAKAYAMA}

The purpose of this short note is to remark that we can state an analog of a famous theorem of Frobeniust on the induced characters of a finite group also for the representations of a general group. $\ddagger$ This extension has not yet been explicitly stated, so far as I know, although it can be quite easily verified.

Let $\mathfrak{g}$ be a group, and let $\mathfrak{h}$ be a subgroup (of a finite or infinite index) of $\mathfrak{g}$.

Definition. Let $F(x)$ and $f(\xi)$ be almost periodic (a. p.) functions (with complex numbers as values) on $\mathfrak{g}$ and $\mathfrak{h}$ respectively. Then we define the compositions of $F(x)$ and $f(\xi)$ by

$$
\begin{aligned}
& f \times F(x)=M_{\xi \in \mathfrak{G}}\left[f(\xi) F\left(\xi^{-1} x\right)\right], \\
& F \times f(x)=M_{\xi \in \mathfrak{G}}\left[F\left(x \xi^{-1}\right) f(\xi)\right] ;
\end{aligned}
$$

where $M_{\xi \in \mathfrak{h}}$ means the construction of the mean with respect to a variable $\xi$ in $\mathfrak{h}$. Here $f \times F(x)$ and $F \times f(x)$ are a. p. functions on $\mathfrak{g}$, and they are linear with respect to both factors, $f(\xi)$ and $F(x)$.

If $\mathfrak{h}_{1}, \mathfrak{h}_{2}, \mathfrak{h}_{3}$ are three subgroups of $\mathfrak{g}$ such that $\mathfrak{h}_{i} \leqq \mathfrak{h}_{k}$ or $\mathfrak{h}_{i} \supseteqq \mathfrak{h}_{k}$ for every $i, k=1,2,3$, then

$$
\left(f_{1} \times f_{2}\right) \times f_{3}=f_{1} \times\left(f_{2} \times f_{3}\right)
$$

for $a$. p. functions $f_{1}\left(\xi_{1}\right), f_{2}\left(\xi_{2}\right), f_{3}\left(\xi_{3}\right)$ on $\mathfrak{h}_{1}, \mathfrak{h}_{2}, \mathfrak{h}_{3}$ respectively.

(Both sides of the equality are a. p. functions on the greatest among the $\mathfrak{h}_{i}$.) This product we denote by $f_{1} \times f_{2} \times f_{3}$.

All these statements we can prove by a procedure similar to that

* Presented to the Society, October 30, 1937.

$\dagger$ G. Frobenius, Ueber Relationen zwischen den Charakteren einer Gruppe und denen ihrer Untergruppen, Berlin Sitzungsberichte, 1898; H. Weyl, Gruppentheorie und Quantenmechanik; J. Levitzki, Ueber vollständig reduzible Ringe und Unterringe, Mathematische Zeitschrift, vol. 33 (1931).

$\ddagger \mathrm{J}$. von Neumann, Almost periodic functions in a group, Transactions of this Society, vol. 36 (1934). Cf. also S. Bochner and J. von Neumann, Almost periodic functions in groups, II, ibid., vol. 37 (1935); W. Maak, Eine neue Definition der fastperiodischen Funktionen, Abhandlungen aus dem Mathematischen Seminar, Hamburg, vol. 11 (1936); B. L. van der Waerden, Gruppen von linearen Transformationen, Ergebnisse der Mathematik, vol. 4 (1935).

By a representation of a group we understand always a bounded one in the field of complex numbers. 
of von Neumann in the paper cited. Therefore we can consider the ring $\Re_{\mathfrak{h}}$ of a. p. functions on $\mathfrak{h}$ as a (right and left) operator-ring of the ring $\Re_{\mathfrak{g}}$ of a. p. functions on $\mathfrak{g}$. If $\mathfrak{M}$ and $\mathfrak{m}$ are submoduli of $\Re_{\mathfrak{g}}$ and $\Re_{\mathfrak{h}}$ respectively, then we denote by $\mathfrak{M} \times \mathfrak{m}$ the submodule of $\Re_{\mathfrak{g}}$ generated by the elements $(F \times f(x), F(x) \in \mathfrak{M}, f(\xi) \epsilon \mathfrak{m})$. We define $\mathfrak{m} \times \mathfrak{M}$ in a similar manner.

Definition. Let $\mathfrak{n}$ be a left ideal of $\Re_{\mathfrak{b}}$ with a finite rank with respect to the field $\Omega$ of complex numbers. ${ }^{*}$ Then $\Re_{\mathfrak{g}} \times \mathfrak{n}$ is obviously a left ideal of $\Re_{\mathfrak{g}}$ (with a finite or infinite rank with respect to $\Omega$ ). We call $\Re_{\mathfrak{g}} \times \mathfrak{n}$ the left ideal of $\Re_{\mathfrak{g}}$ induced by $\mathfrak{n}$.

As is well known, there is an idempotent element $c(\xi)$ in $\Re_{\mathfrak{b}}$ such that $\mathfrak{n}=\Re_{\mathfrak{h}} \times c$; it is $f \times c=f$ for every $f(\xi)$ in $\mathfrak{n}$. If $F(x) \epsilon \Re_{\mathfrak{g}}$ and $f(\xi) \in \mathfrak{n}$, then we have $F \times f=F \times(f \times c)=(F \times f) \times c \epsilon \Re_{\mathfrak{g}} \times c$. Therefore $\Re_{\mathfrak{g}} \times \mathfrak{n}$ $\leqq \Re_{\mathfrak{g}} \times c$, and this implies $\Re_{\mathfrak{g}} \times \mathfrak{n}=\Re_{\mathfrak{g}} \times c$.

The ideal $\Re_{\mathfrak{g}} \times \mathfrak{n}$ consists of all functions $G(x) \epsilon \Re_{\mathfrak{g}}$ such that for every $x \in \mathfrak{g}$ the function $G(x \xi)$ of $\xi \in \mathfrak{h}$ lies in $\mathfrak{n}$.

Let $G(x)$ have the property stated above. Then

in particular,

$$
M_{\eta \in \mathfrak{\xi}}\left[G\left(x \xi \eta^{-1}\right) c(\eta)\right]=G(x \xi) ;
$$

$$
G \times c(x)=M_{\eta \mathfrak{h}}\left[G\left(x \eta^{-1}\right) c(\eta)\right]=G(x)
$$

that is, $G(x) \epsilon \Re_{\mathfrak{g}} \times c=\Re_{\mathfrak{g}} \times \mathfrak{n}$.

The other half of the statement is obvious.

Now we have the following theorem:

TheOREM. Let $\mathfrak{n}$ be a minimal left ideal of $\Re_{\mathfrak{h}}$, and $\mathfrak{b}$ the irreducible representation of $\mathfrak{h}$ defined by $\mathfrak{n}$. Let $\mathfrak{D}$ be an irreducible representation of $\mathfrak{g}$, and $\mathfrak{S}$ the two-sided ideal of $\Re_{\mathfrak{g}}$ belonging to $\mathfrak{D} . \dagger$ We denote by $\mathfrak{D}(\mathfrak{h})$ a representation of $\mathfrak{h}$ formed by the matrices in $\mathfrak{D}$ which correspond to the elements of $\mathfrak{h}$. If the number of the irreducible constituents of $\mathfrak{D}(\mathfrak{h})$ equivalent to $\mathfrak{b}$ is $g$, then the representation of $\mathrm{g}$ defined by the $\widetilde{S}$-component $\mathfrak{S} \times \mathfrak{N}=\mathfrak{S} \times \mathfrak{n}$ of the induced left ideal $\mathfrak{N}=\Re_{\mathfrak{g}} \times \mathfrak{n}$ consists of just $\mathrm{g}$ irreducible constituents (equivalent to $\mathfrak{D})$.

Proof. $\ddagger$ Let $E(x)$ denote the principal unit of $\mathfrak{S}$, and put

* Note that a submodule of $\Re_{\mathfrak{h}}\left(\Re_{\mathfrak{g}}\right)$ with a finite rank with respect to $\Omega$ is a left ideal if and only if it is an $\mathfrak{h}$-( $\mathfrak{g}$-)left-module; where we define the multiplication of $\alpha \in \mathfrak{h}$ and $f(\xi) \in \Re_{\mathfrak{h}},\left(a \epsilon_{\mathfrak{g}}\right.$ and $\left.F(x) \in \Re_{\mathfrak{g}}\right)$, by $\alpha \cdot f(\xi)=f\left(\alpha^{-1} \xi\right),\left(a \cdot F(x)=F\left(a^{-1} x\right)\right)$. Then we have $(\alpha \cdot f) \times F=\alpha \cdot(f \times F),(a \cdot F) \times f=a \cdot(F \times f)$, and so on.

$\dagger$ Linear aggregates of the matric elements of $\mathfrak{D}$ form a two sided ideal of $\Re_{\mathfrak{g}}$, which is isomorphic to a matric ring.

$\ddagger$ The following proof is only a slight modification of the proof in Weyl, loc. cit. 


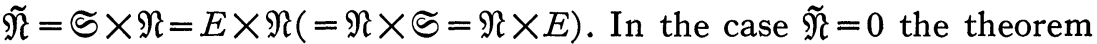
is obvious. We assume therefore $\tilde{\mathfrak{R}} \neq 0$, that is, $c \times E \neq 0$.

If we denote the degree of the representation $\delta$ by $r$, then the twosided ideal $\mathfrak{z}$ of $\Re_{\mathfrak{b}}$ belonging to $\mathfrak{b}$ is a direct sum of $r$ minimal left ideals operator-isomorphic to $\mathfrak{n}$ :

$$
\mathfrak{z}=\mathfrak{n}^{(1)}+\mathfrak{n}^{(2)}+\cdots+\mathfrak{n}^{(r)}, \quad \mathfrak{n}^{(i)} \cong \mathfrak{n} .
$$

Put $\mathfrak{N}^{(i)}=\Re_{\mathfrak{g}} \times \mathfrak{n}^{(i)}$ and $\tilde{\mathfrak{N}}^{(i)}=\mathfrak{S} \times \mathfrak{N}^{(i)}=\mathfrak{S} \times \mathfrak{n}^{(i)}$. It is easy to see that $\mathfrak{R}_{\mathfrak{g}} \times \mathbb{B}$ is the direct sum $\mathfrak{N}^{(1)}+\mathfrak{N}^{(2)}+\cdots+\mathfrak{N}^{(r)}$, and also that $\mathfrak{S} \times \Re_{\mathfrak{g}} \times \mathbb{z}=\mathfrak{S} \times \mathbb{z}=\tilde{\mathfrak{N}}^{(1)}+\tilde{\mathfrak{N}}^{(2)}+\cdots+\tilde{\mathfrak{N}}^{(r)}$. Now suppose that $\tilde{\mathfrak{R}}$ is a direct sum of $h$ minimal left ideals. (Our purpose is to show $h=g$.) Then each of $\tilde{\mathfrak{N}}^{(i)}$ has the same property: $\tilde{\mathfrak{N}}^{(i)}=\mathfrak{R}_{1}^{(i)}+\mathfrak{R}_{2}{ }^{(i)}+\cdots+\mathfrak{R}_{h}{ }^{(i)}$, for it is operator-isomorphic to $\tilde{\mathfrak{R}}$.

Let $e(\xi)$ be the principal unit of $\xi$. We have $\mathfrak{S} \times \mathfrak{g}=\Re_{\mathfrak{g}} \times(e \times E)$ and $e \times E=E \times e \times E=E \times e, \quad(e \times E)^{2}=E \times e \times E=e \times E$.

Moreover

$$
\mathfrak{S}=\mathfrak{S} \times \mathfrak{B}+\mathfrak{R}^{*}=\mathfrak{R}_{1}^{(1)}+\mathfrak{R}_{2}^{(1)}+\cdots+\mathfrak{R}_{h}^{(r)}+\mathfrak{R}^{*}
$$

for a suitably chosen left ideal $\mathfrak{\imath}^{*}$ of $\mathfrak{\subseteq}$. From this decomposition we see in the usual manner that $(e \times E) \times \Re_{\mathfrak{g}} \times(e \times E)$ is a matric ring of degree $r h$ (over $\Omega$ ):

$$
\begin{gathered}
(e \times E) \times \Re_{\mathfrak{g}} \times(e \times E)=\sum_{\substack{k, i=1, \ldots, r \\
\mu, \lambda=1, \cdots, h}} C_{\mu \lambda}^{(k)(i)} \Omega, \\
(e \times E) \times \mathfrak{R}_{\lambda}^{(i)}=\sum_{k, \mu} C_{\mu \lambda}^{(k)(i)} \Omega,
\end{gathered}
$$

$\left(C_{\mu \lambda}^{(k)(i)}(x)\right.$ being matric units). Here $(e \times E) \times \Omega_{\lambda}^{(i)}=e \times\left(E \times \mathbb{R}_{\lambda}^{(i)}\right)$ $=e \times \Omega_{\lambda}{ }^{(i)}$, and therefore $\left(e \times \Omega_{\lambda}{ }^{(i)}: \Omega\right)=r h$.

On the other hand $\mathfrak{R}_{\lambda}{ }^{(i)}$ is, considered as an $\mathfrak{h}$-left-module, completely reducible, for it defines a representation of $\mathfrak{h}$ equivalent to $\mathfrak{D}(\mathfrak{h})$. Let $\mathfrak{R}_{\lambda}^{(i)}=\mathfrak{M}_{1}+\mathfrak{M}_{2}+\cdots+\mathfrak{M}_{l}$ be its decomposition into simple (minimal) submoduli. According to our assumption just $g$ of $\mathfrak{M}_{j}$ are operator-isomorphic to $\mathfrak{n}$ with respect to $\mathfrak{h}$, and therefore also with respect to $\Re_{\mathfrak{b}} \dagger$ This implies $\left(e \times \Re_{\lambda}{ }^{(i)}: \Omega\right)=r g$.

Comparing with the above result, we obtain $h=g$.

\section{The Institute for Advanced Study}

$\dagger$ A submodule, with a finite rank, of $\Re_{\mathfrak{g}}$ is an $\Re_{\mathfrak{h}}$-left-module if and only if it is an $\mathfrak{h}$-left-module. Two such moduli are operator-isomorphic with respect to $\Re_{\mathfrak{h}}$ if and only if they are so with respect to $\mathfrak{h}$. The same holds for the isomorphism between such a module and a left ideal of $\Re_{\mathfrak{b}}$. 